Please do not remove this page

RMIT

UNIVERSITY

\title{
Performance of variable step-size dithered signed error CMA for blind equalization
}

Jusak, Jusak; Hussain, Zahir; Harris, Richard

https://researchrepository.rmit.edu.au/esploro/outputs/9921858206901341/filesAndLinks?institution=61RMIT_INST\&index=null

Jusak, J., Hussain, Z., \& Harris, R. (2004). Performance of variable step-size dithered signed error CMA for blind equalization. IEEE TENCON 2004 - Analog and Digital Techniques in Electrical Engineering, 684-687. https://doi.org/10.1109/TENCON.2004.1414688

Published Version: https://doi.org/10.1109/TENCON.2004.1414688

Repository homepage: https://researchrepository.rmit.edu.au

(c) 2004 IEEE. Personal use of this material is permitted. However, permission to reprint/republish this material for advertising or promotional purposes or for creating new collective works for resale or redistribution to servers or lists, or to reuse any copyrighted component of this work in other works must be obtained from the IEEE.

Downloaded On 2023/04/26 22:28:26 +1000 


\title{
PERFORMANCE OF VARIABLE STEP-SIZE DITHERED SIGNED-ERROR CMA FOR BLIND EQUALIZATION
}

\author{
Jusak Jusak, Zahir M. Hussain and Richard J. Harris \\ Centre for Advanced Technology in Telecommunications (CATT) \\ School of Electrical and Computer Engineering \\ RMIT University, Melbourne, Victoria 3000, Australia \\ E-mail: s3001898@student.rmit.edu.au,zmhussain@ieee.org, richard@catt.rmit.edu.au
}

\begin{abstract}
Recently a dithered signed-error constant modulus algorithm (DSE-CMA) has been proposed, associated with fractionally-spaced equalization, for the purpose of low complexity implementation of constant modulus algorithm (CMA). DSE-CMA has robustness properties closely resembling those of CMA under certain restrictions. As the CMA is slow in achieving its minimum mean squared error, so is the DSE-CMA. In this work, we apply an adaptive step-size instead of a fixed one, and then examine the performance of few variable step-size algorithms that result in faster convergence while preserve the low computational complexity and robustness properties of the DSE-CMA algorithm. We also derive the excess mean-squared error in the case of noisy channel to examine the robustness of the algorithms.
\end{abstract}

\section{INTRODUCTION}

High-speed data communication over a bandlimited channel is subject to Inter-Symbol Interference (ISI) as a result of transmitter receiver filtering and multipath propagation. Mitigation of such kind of distortion calls for the use of equalization filter[1]. In the last few years, blind equalization techniques have gained an increasing interest. The most popular and implemented blind adaptation algorithm is the constant modulus algorithm (CMA)[2] and [3].

Recent studies (associated with the fractionallyspaced equalization constant modulus (FSE-CM) algorithm) have developed the dithered signed-error constant modulus algorithm, which has been proved to bear a resemblance to those of CMA properties when some restrictions are satisfied [4]. The only drawback of DSE-CMA compared to original form of CMA is the increment of excess mean-squared error (EMSE). Due to multi-modality of DSE-CMA cost surface, the convergence rate is greatly affected by initialization of the equalizer. Our studies in [6] show that DSE-CMA is slow in convergence compared to the original form of CMA when the algorithm is initialized with a large value of $\|\mathbf{f}\|$, where $\mathbf{f}$ is the equalizer coefficients vector.

0-7803-8560-8/04/\$20.00(c)2004IEEE
As it has been pointed out in [5], CMA has desirable robustness properties similar to those of LMS under perfect blind equalizability (PBE) condition. Thus, it is also worthwhile to evaluate the variable step-size scheme (that was previously applied to the LMS algorithm) to the DSE-CMA with expectation to increase the convergence rate as compared to the fixed step-size algorithm. On the other hand, it has been derived in [4] that the final excess mean square error (EMSE) is directly proportional to the adaptation step-size of the DSE-CMA, while the convergence time increases as the step-size decreases. In this paper, we will examine the use of a family of variable step-size adaptation based on the prediction error [7] and [8] to cope with the slow convergence rate in the DSE-CMA. We also present a method to adapt the variable step-size by utilizing an averaging of the prediction error. The idea of using prediction error to control the step-size adaptation is motivated by the fact that a large prediction error will increase the step-size in order to provide faster tracking, while a small prediction error will decrease the step-size in order to give smaller misadjustment. The final aim is to increase the convergence speed of DSE-CMA while at the same time maintaining the robustness properties and low computational complexity of the algorithm.

\section{SYSTEM MODEL}

For space limitations we focus on the case of equalizing communication systems with an FIR channel model and fractionally-spaced filters with sampling interval $T / 2$ ( $T$ being the symbol period). A multirate model of a digital communication system is shown in Fig. 1, where the source symbol $\left(x_{n}\right)$ is drawn from a finite alphabet; it is a random variable with zero mean, independent and identically distributed (i.i.d) with variance $\sigma_{x}^{2}=E\left\{\left|x_{n}\right|^{2}\right\}$, baud-spaced at sample index $n$, while the fractionally-spaced sample is denoted using the sample index $k$. The vector $\mathbf{c}=\left[c_{0}, \cdots, c_{N_{c}-1}\right]^{t}$ is an $N_{c} \times 1$ vector representing the fractionally-spaced channel impulse response and $\mathbf{f}=\left[f_{0}, \cdots, f_{N_{f}-1}\right]^{t}$ is a fractionally-spaced equalizer coefficient vector of size $N_{f} \times 1$. The number of channel and equalizer coefficients is denoted as $N_{c}$ and $N_{f}$, respectively. The fractionally-spaced received signal is denoted as $r_{k}$. Note that the original source 


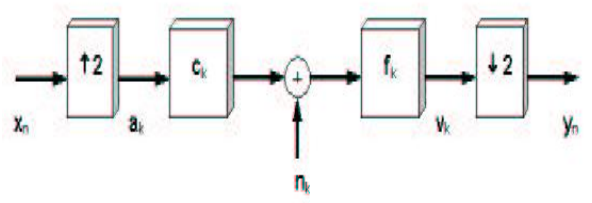

Fig. 1. Multirate model for noiseless fractionallyspaced systems.

symbol is $x(n)=\left[x_{n}, x_{n-1}, \cdots, x_{n-N_{x}+1}\right]^{t}$, which is a vector of length $N_{x}=\left\lfloor\left(N_{c}+N_{f}-1\right) / 2\right\rfloor$ with baud-spaced source symbols.

Defining $\mathbf{C}$ as the time decimated channel convolution matrix of $N_{x} \times N_{f}$, defined by:

$$
\left[\begin{array}{ccccccc}
c_{1} & c_{0} & & & & & \\
\vdots & \vdots & c_{1} & c_{0} & & & \\
c_{N_{c}-1} & c_{N_{c}-2} & \vdots & \vdots & \ddots & c_{1} & c_{0} \\
& & c_{N_{c}-1} & c_{N_{c}-2} & \ddots & \vdots & \vdots \\
& & & & & c_{N_{c}-1} & c_{N_{c}-2}
\end{array}\right]
$$

the system output may be expressed as:

$$
y_{n}=\mathbf{x}^{t}(n) \mathbf{C f},
$$

where $(.)^{t}$ represents a transpose operator. The equalizer coefficients are updated according to the following algorithm:

$$
\mathbf{f}(n+1)=\mathbf{f}(n)+\mu \mathbf{r}^{*}(n) \psi_{\mathrm{cma}}\left(y_{n}\right),
$$

where $\mu$ is a constant called the step-size (usually small), and $\psi_{\mathrm{cma}}\left(y_{n}\right)$ is the CMA error function.

The error function of CMA is described as [2]:

$$
\psi_{\mathrm{cma}}\left(y_{n}\right)=y_{n}\left(\gamma-\left|y_{n}\right|^{2}\right) \text {, }
$$

where $\gamma$ is a dispersion constant, which is defined as $\gamma=E\left[|x(n)|^{4}\right] / E\left[|x(n)|^{2}\right]$.

Under a perfect blind equalization (PBE) condition (as stated in [5]), equalizers minimizing the CM cost function can perfectly recover the original source symbols for some system delay $\delta$ (i.e., $y_{n}=x_{n-\delta}$ ). Requirements for perfect symbol recovery conditions are: in the $T / 2$-spaced fractionally-spaced and a channel with even-length, a necessary condition for channel invertibility is that $N_{f} \geq N_{c}-2$, no additive channel noise, for the even-length $T / 2$-spaced channel impulse response, $c_{\text {even }}\left(z^{-1}\right)$ and $c_{\text {odd }}\left(z^{-1}\right)$ have no common roots, sub-Gaussian source and i.i.d. zero mean source (white source).

By transforming the error function in eq. (4) into a sign using a sign function, complexity reduction in the adaptation process (3) is achieved, as multiplication is now reduced to a sign operation. However, in order to preserve information lost in the quantization process, a small perturbation random signal is added, so-called dithering technique [4]. Thus the error function in eq. (4) becomes:

$$
\psi_{\mathrm{dse}-\mathrm{cma}}\left(y_{n}\right)=\alpha \operatorname{sign}\left(y_{n}\left(\gamma-\left|y_{n}\right|^{2}\right)+\alpha d_{n}\right),
$$

where $\alpha$ is a dither amplitude and $\left\{d_{n}\right\}$ is an i.i.d process uniformly distributed on $(-1,1]$.

\section{VARIABLE STEP-SIZE DSE-CMAS}

As expressed in eq. (3), the choice of the step-size $\mu$ reflects a tradeoff between misadjustment and the speed of adaptation. The aim of using variable step-size in adaptation algorithms is to provide capability of giving both fast tracking and small misadjustment at the same time.

The variable step-size algorithm for adjusting the step-size $\mu$ may be controlled by the prediction error. It was shown that this algorithm outperforms the fixed step-size adaptation in LMS type algorithms [7] and [8]. Based on the similarity in the desirable robustness properties between LMS and CMA algorithms, we will utilize the variable step-size to replace the constant step-size for the conventional DSECMA adaptation algorithm in eq. (5). However, we need to redefine the estimation error $\epsilon$ in [7] according to the error function of blind DSE-CMA, where $\epsilon=\psi_{\mathrm{dse}-\mathrm{cma}}\left(y_{n}\right)$. Instead of choosing $\epsilon$ as a function of $\psi_{\mathrm{cma}}\left(y_{n}\right)$ as in our previous work [9], it can be justified based on DSE-CMA properties that determining $\epsilon$ as a function of $\psi_{\mathrm{dse}-\mathrm{cma}}\left(y_{n}\right)$ will guarantee the update identical to the CMA. Those properties can be summarized as follows: $\alpha$ is selected large enough to satisfy $\alpha \geq\left|\psi_{\mathrm{cma}}\left(y_{n}\right)\right|$. The initialization and, thus, adaptation is performed within the convex hull $\mathcal{F}_{\alpha}$ which is formed by the hyperplanes $\mathcal{B}_{\alpha}:=$ $\left\{\mathbf{f}:\left|\mathbf{r}^{t} \mathbf{f}\right|=\psi_{\text {cma }}^{-1}(\alpha)\right.$ for $\left.\mathbf{r} \in \mathcal{R}\right\} . \mathcal{R}$ is the set of all possible received vector $\mathbf{r}$. The steady state excess mean-squared error DSE-CMA ( $\left.J_{\mathrm{ex}(\mathrm{dse}-\mathrm{cma})}\right)$ should be chosen to satisfy $J_{\mathrm{ex}(\mathrm{dse}-\mathrm{cma})}=K(\alpha) J_{\mathrm{ex}(\mathrm{cma})}$, where $K(\alpha):=\frac{\alpha^{2}}{E\left\{x_{n}^{6}\right\}-\kappa_{x}^{2} \sigma_{x}^{2}}, \kappa_{x}$ and $\sigma_{x}^{2}$ are the kurtosis and the second moment of the source symbol, respectively.

\subsection{Algorithms}

Adaptation of DSE-CMA is followed by adaptation of the step-size $\mu(n)$ according to the following schemes: Type 1:

$$
\mu(n)=\lambda \mu(n-1)+\beta \epsilon^{2},
$$

with $0<\lambda<1, \beta>0$, and

$$
\mu(n)= \begin{cases}\mu_{\max }, & \text { for } \mu(n)>\mu_{\max }, \\ \mu_{\min }, & \text { for } \mu(n)<\mu_{\min }, \\ \mu(n), & \text { otherwise. }\end{cases}
$$

Adaptation step-size in Type 1 is based on the energy of instantaneous error, where the constant parameter $\beta$ controls the level of misadjustment as well as the convergence time of the algorithm. The constant value $\mu_{\max }$ is selected to provide maximum possible convergence speed, i.e., near to the point where the algorithm is going to be unstable. The constant $\mu_{\min }$ is chosen to provide a minimum level of tracking ability. The more 


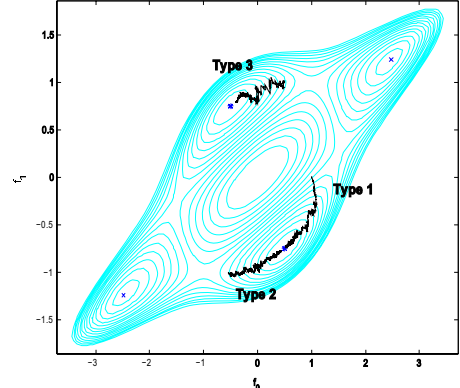

Fig. 2. Trajectories of variable step-size DSE-CMA algorithms for BPSK, well-behaved noisy channel with $\mathrm{SNR}=30 \mathrm{~dB}$. Global MSE minima marked by "*". Local MSE minima marked by " $x$ ".

realistic approach of Type 1 is using an average value of the prediction error as in Type 2 below.

Type 2:

$$
\mu(n+1)=\lambda \mu(n)+\beta p(n)^{2},
$$

where the variable $p(n)$ is updated according to the average value of the squared error as follows:

$$
p(n)=\eta p(n-1)+(1-\eta) \epsilon(n-1)^{2},
$$

with $0<\eta<1$, which is usually called as the forgetting factor. The constant value $\lambda$ and $\beta$ are the same as those of the Type 1 algorithm.

Type 3:

$$
\mu(n+1)=\lambda \mu(n)+\beta p(n)^{2},
$$

where the variable $p(n)$ is updated according to the autocorrelation error as follows:

$$
p(n)=\eta p(n-1)+(1-\eta) \epsilon(n) \epsilon(n-1),
$$

with $0<\eta<1$, which is also called as the forgetting factor.

The mean behaviour and stability analysis of the algorithms have been discussed extensively in [7] and [8]. The trajectory of the variable step-size DSECMA for different types of schemes can be seen in Fig. 2. In this figure the constant modulus cost surface has been plotted for a well-behaved channel [5] $\mathbf{c}=[1.0,-0.5,0.2,0.3]$ with BPSK source signal, and $\mathrm{SNR}=30 \mathrm{~dB}$.

\subsection{The excess MSE of DSE-CMA in noisy channel}

When the additive white Gaussian noise perturbs the channel, the vector of the received signal (z) becomes:

$$
\mathbf{z}=\mathbf{r}+\mathbf{w}
$$

where $\mathbf{w}$ is the vector of additive white Gaussian noise.

Following the assumption in [4], excess MSE is defined at the time index $n$ as the expected error, which is achieved by the zero-forcing solution $\left(\mathbf{f}_{\delta}\right)$. Using the parameter error vector $\mathbf{f}=\mathbf{f}-$ $\mathbf{f}_{\delta}$, the output error can be defined as $e_{n}=\mathbf{r}^{t} \tilde{\mathbf{f}}+$ $\mathbf{w}^{t} \tilde{\mathbf{f}} . \quad$ It is assumed that $\mathbf{F}(n)=E\left\{\mathbf{f}(n) \tilde{\mathbf{f}}^{t}(n)\right\}$. The source-power-normalized autocorrelation matrix $\mathbf{R}=\frac{1}{\sigma_{x}^{2}} E\left\{\mathbf{r}(n) \mathbf{r}^{t}(n)\right\}$ is Toeplitz, therefore it can be equated to $\mathbf{R}=\mathbf{Q} \Lambda \mathbf{Q}^{t}$ to simplify the derivation, where the matrix $\Lambda$ is diagonal and the matrix $\mathbf{Q}$ is orthogonal. Applying the same way to $\mathbf{F}(n)$ yields $\mathbf{F}(n)=\mathbf{Q X}(n) \mathbf{Q}^{t}$. On the other hand, if the PBE condition is hold, than it is guaranteed that $\tilde{\mathbf{f}}$ will achieves zero error. Hence, the small-error approximation approach is also held. The excess MSE can be expressed as follows:

$$
J_{\mathrm{ex}}=\lim _{n \rightarrow \infty} J_{\mathrm{ex}}(n),
$$

where

$$
\begin{aligned}
J_{\mathrm{ex}}(n) & =E\left\{\left|e_{n}\right|^{2}\right\} \\
& =E\left\{\left|\mathbf{r}^{T} \tilde{\mathbf{f}}+\mathbf{w}^{T} \tilde{\mathbf{f}}\right|^{2}\right\} .
\end{aligned}
$$

Here, it is assumed that error vector $\tilde{\mathbf{f}}$ is statistically independent of the input vector $\mathbf{r}$ and noise vector $\mathbf{w}$. The step-size is chosen small enough.

Following the procedure in [4] and assuming that white Gaussian noise is added, $J_{\text {ex }}$ may be expressed as follows:

$$
J_{\mathrm{ex}}=\sigma_{s}^{2} \sum_{i} \lambda_{i} x_{i}(n)+\sigma_{w}^{2} \Sigma_{i} x_{i}(n),
$$

where $\lambda_{i}$ and $x_{i}$ is the $i^{\text {th }}$ diagonal element of $\Lambda$ and $\mathbf{X}(n)$, respectively. $\sigma_{w}^{2}$ is the second moment of Gaussian noise. It is clear that eq.(15) shows the increment of excess MSE because of addition white Gaussian noise to the channel.

\section{SIMULATION RESULTS}

We examined the variable step-size DSE-CMAs in different environments. We have simulated a BPSK source signal under noiseless channel, a BPSK source signal under noisy channel (to show the effect of noise on the variable step-size algorithms), and an 8-PAM source signal (to show the effect of higher-order modulation on the variable step-size algorithms). Results have been obtained via generation of independent symbols using 100 different runs. In all simulations, the parameters of the algorithms have been set in order to achieve the same minimum mean-squared error (MMSE), so that the convergence rate can be compared easily. For all variable step-size algorithms, we used $\mu_{\max }=0.02$ and $\mu_{\min }=1 \times 10^{-5}$. The impulse response response of the channel is: $\mathbf{c}=$ $[0.04,-0.05,0.07,-0.21,-0.5,0.72,0.36,0.21$, $0.03,0.07]$.

The performance of the variable step-size DSECMA has been compared to that of the fixed step-size DSE-CMA. It can be seen in Fig. 3 that variable stepsize algorithms in noiseless channel outperform the fixed step-size DSE-CMA in achieving its convergence point. For the all variable step-size algorithms, $\lambda$ has been set to 0.97 to provide a forgetting factor, while $\beta$ in Type 1 algorithm is set to $2 \times 10^{-3}, 9 \times 10^{-3}$ in 


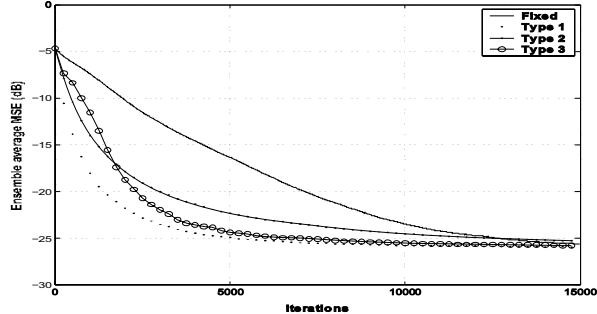

Fig. 3. Averaged MSE trajectories for fixed stepsize DSE-CMA and variable step-size DSE-CMAs for noiseless channel. Source signal is modulated with BPSK.

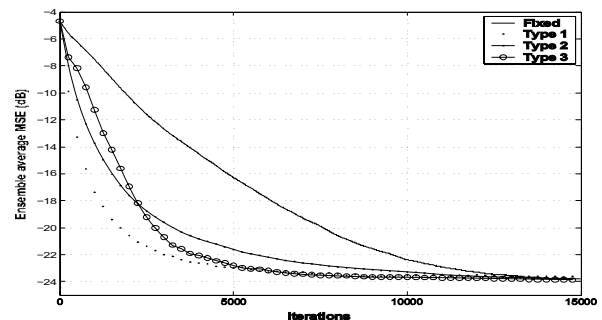

Fig. 4. Averaged MSE trajectories for fixed stepsize DSE-CMA and variable step-size DSE-CMAs for noisy channel, with $\mathrm{SNR}=30 \mathrm{~dB}$. Source signal is modulated with BPSK.

Type 2 algorithm, and $5 \times 10^{-2}$ for the third algorithm in order to achieve the MMSE $-26 \mathrm{~dB}$. The parameter $\eta$ in Type 2 and Type 3 algorithms was set to 0.99 , while the fixed step-size of the DSE-CMA was chosen as $1 \times 10^{-3}$ and $\alpha=1$. The source signal of BPSK has been used in this simulation.

$A$ violation of the $\mathrm{PBE}$ condition is considered where Gaussian noise with $\mathrm{SNR}=30 \mathrm{~dB}$ is added. Fig. 4 shows that the variable step-size DSE-CMA is still able to achieve its convergence point. As compared to the fixed step-size DSE-CMA, it can be seen that variable step-size DSE-CMAs have faster convergence. The setting of parameters for all algorithms is the same as in the previous simulation. However, it is clear that MMSE is now degraded to $-24 \mathrm{~dB}$ as a result of noise addition.

Secondly, we consider a source signal modulated with 8-PAM. The parameters of the algorithms were set to achieve a steady state MMSE of $-26 \mathrm{~dB}$. Fig. 5 shows that when the higher constellation of the modulation is used, the performance of the variable stepsize algorithms in terms of convergence rate decreases. Yet, in overall the convergence rate of variable stepsize DSE-CMA is faster than the fixed step-size DSECMA. The parameters were set as follows: $\lambda=0.97$ for all algorithms, $\beta=5 \times 10^{-5}$ for Type 1 algorithm, $\beta=6 \times 10^{-5}$ for Type 2 algorithm and $\beta=2 \times 10^{-4}$ for Type 3 algorithm, while $\eta=0.99$ for both Type 2 and Type 3 algorithms. The fixed step-size of the DSE-CMA was chosen as $1 \times 10^{-3}$ and $\alpha=1.5$

\section{CONCLUSIONS}

In this paper, we proposed a dithered signed-error constant modulus algorithm (DSE-CMA) with variable

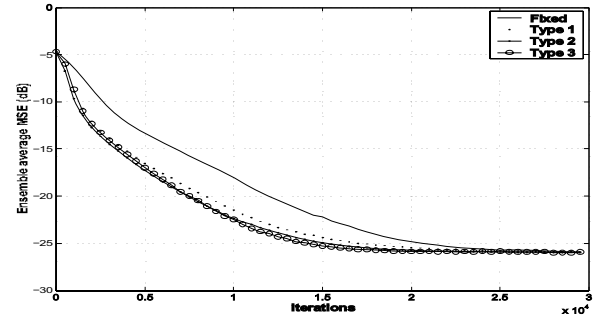

Fig. 5. Averaged MSE trajectories for fixed stepsize DSE-CMA and variable step-size DSE-CMAs for noiseless channel. Source signal is modulated with 8PAM.

step-size. Different variable step-size schemes based on prediction error has been examined. It is shown that the variable step-size DSE-CMA algorithms are all robust against violation of the perfect blind equalization (PBE) condition, where in this simulation Gaussian noise has been added. Thus, together with its low computational complexity properties, variable stepsize DSE-CMA can yield a higher performance for blind adaptive channel equalization than the fixed stepsize DSE-CMA algorithm.

\section{REFERENCES}

[1] J. G. Proakis, Digital Communications, 3ed, McGraw Hill International, New York, 1995.

[2] D. Godard, "Self-recovering equalization and carrier tracking in two dimensional data communication systems," IEEE Trans. Commun, 28, pp. 1867-1875, 1980.

[3] J. R. Treichler and B. G. Agee, "A new approach to multipath correction of contant modulus signals," IEEE Trans. Acoust. Speech. Signal Processing, ASSP-31, 2, pp. 459-472, 1983.

[4] P. Schniter and R. J. Johnson, Jr., "Dithered signederror: robust, computationally efficient blind adaptive equalization," IEEE Trans. Signal Processing, 47, 6 , pp. 1592-1603, Jun 1999.

[5] C. R. Johnson, Jr., P. Schniter, T. J. Endres, J. D. Behm, D. R. Brown and R. A. Casas, "Blind equalization using the constant modulus criterion: A review," Proc. IEEE, 86, 10, pp. 1927-1950, Dec 2003.

[6] J. Jusak, Z.M. Hussain and R.J. Harris, "Direct Adaptive Blind Linear Equalization over Fading Multipath Channels," Proc. ATNAC 2003 Australian Telecommunication, Network and Applications Conference, Dec 2003.

[7] R. Kwong and E. Johnston, "A Variable Step Size LMS Algorithm," IEEE Trans. Signal Processing, 40, 7, pp. 1633-1642, Jul 1992.

[8] T. Aboulnasr, and K. Mayyas, "A Robust Variable stepsize LMS-type algorithm: analysis and simulations," IEEE Trans. Signal Processing, 45, 3, pp. 631-639, Mar 1997.

[9] J. Jusak, Z. M. Hussain and R. J. Harris, "A High Performance Blind Adaptive Filtering Algorithm," Proc. 2nd WSEAS International Conference on Electronics, Control and Signal Processing (ICECS '03), Dec 2003. 